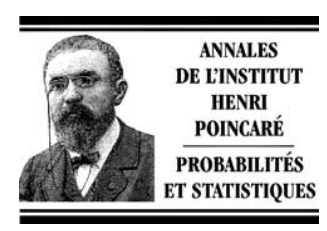

www.elsevier.com/locate/anihpb

\title{
Real harmonizable multifractional Lévy motions
}

\author{
Céline Lacaux \\ Université Paul Sabatier UFR MIG, laboratoire de statistique et de probabilités, 118, route de Narbonne, 31062 Toulouse, France
}

Received 23 June 2002; received in revised form 22 July 2003; accepted 5 November 2003

Available online 10 February 2004

\begin{abstract}
In this paper, the class of real harmonizable multifractional Lévy motions (in short RHMLMs) is introduced. This class is a generalization of the multifractional Brownian motion (in short MBM) and of the class of real harmonizable fractional Lévy motions. One of its main interest is that it contains some non-Gaussian fields which share many properties with the MBM. RHMLMs have locally Hölder sample paths and their Hölder exponent is allowed to vary along the trajectory. Moreover these fields are locally asymptotically self-similar. The multifractional function can be estimated with the localized generalized quadratic variations.
\end{abstract}

C 2004 Elsevier SAS. All rights reserved.

\section{Résumé}

Cet article introduit une classe de champs réels appelés champs de Lévy multifractionnaires au moyen d'une représentation harmonisable. Cette classe contient à la fois celle des champs de Lévy fractionnaires et le mouvement Brownien multifractionnaire (MBM en abrégé). Elle fournit notamment des exemples de champs non gaussiens ayant des propriétés semblables à celles du MBM. En particulier, les trajectoires d'un champ de Lévy multifractionnaire sont presque sûrement localement höldériennes. Par ailleurs, l'exposant de Hölder ponctuel peut varier le long d'une trajectoire et est égal à celui trouvé dans le cas du MBM. D'autre part, ces champs sont aussi localement asymptotiquement autosimilaires. Enfin l'étude est complétée par l'identification de la fonction multifractionnaire au moyen des variations quadratiques locales et généralisées. (c) 2004 Elsevier SAS. All rights reserved.

MSC: 60G17; 62G05

Keywords: Local asymptotic self-similarity; Multifractional function; Identification

Mots-clés : Autosimilarité locale; Fonction multifractionnaire ; Identification

\section{Introduction}

The fractional Brownian motion (in short FBM), introduced by Mandelbrot and Van Ness in [12], provides a powerful model in applied mathematics. The FBM of index $H(0<H<1)$ is the only centered Gaussian field, vanishing at zero, with stationary increments and which is self-similar with index $H$. Its Hurst exponent governs all

E-mail address: lacaux@1sp.ups-tlse.fr (C. Lacaux).

0246-0203/\$ - see front matter (C) 2004 Elsevier SAS. All rights reserved.

doi:10.1016/j.anihpb.2003.11.001 
its properties. As an example, the pointwise Hölder exponent of $B_{H}$ is almost surely equal to $H$. However the field of applications is restricted because the FBM is a Gaussian field. Moreover since the pointwise Hölder exponent of $B_{H}$ is almost surely constant, the FBM can not be used to model some phenomena for which regularity varies in space.

In order to extend the range of applications, the multifractional Brownian motion (in short MBM) has been introduced independently in $[13,8]$. This Gaussian field does not have any more stationary increments. Moreover the MBM does not remain self-similar but is locally asymptotically self-similar (in short lass). Let us recall how a lass field is defined in [8]. A field $(X(u))_{u \in \mathbb{R}^{d}}$ is locally asymptotically self-similar with multifractional function $h$ if for all $x \in \mathbb{R}^{d}$,

$$
\lim _{\lambda \rightarrow 0^{+}}\left(\frac{X(x+\lambda u)-X(x)}{\lambda^{h(x)}}\right)_{u \in \mathbb{R}^{d}} \stackrel{(d)}{=}(T(u))_{u \in \mathbb{R}^{d}},
$$

where the non-degenerate field $(T(u))_{u \in \mathbb{R}^{d}}$ is called the tangent field at point $x$. Furthermore the Hölder exponent of the MBM is allowed to vary along the trajectory. Then the MBM is used as a toy-model for modeling mountains because it allows to take into account erosion phenomena. Ayache and Lévy Véhel (see [2]) have generalized the MBM in order to have more irregularity. Their fields remain lass Gaussian fields, like those studied in [4,5]. Nevertheless in practice, one often observes non-Gaussian phenomena, see for instance [11,16] for image modeling, and thus can not use these models. Real harmonizable fractional Lévy motions (in short RHFLMs), introduced by Benassi, Cohen and Istas in [6], make up a class of lass fields which includes non-Gaussian fields and the FBM. However their increments are stationary and their Hölder exponent is almost surely equal to a constant.

The main aim of this paper is to introduce some non-Gaussian lass fields whose Hölder exponent varies along the trajectory. These fields will be called real harmonizable multifractional Lévy motions (in short RHMLMs).

Let us recall that a RHFLM $X_{H}$ of index $H(0<H<1)$ is defined as the stochastic integral:

$$
X_{H}(x)=\int_{\mathbb{R}^{d}} \frac{\mathrm{e}^{-i x \cdot \xi}-1}{\|\xi\|^{d / 2+H}} L(d \xi)
$$

where $\|\xi\|$ is the Euclidean norm of $\xi$ and $L(d \xi)$ is a Lévy random measure that has moments of every order. Then RHMLMs are defined by substituting in (1) to the constant parameter $H$ a locally Hölder function $h$. When $L(d \xi)$ is a Brownian measure $W(d \xi)$, one obtains the harmonizable representation of the MBM.

Moreover, in order to identify the multifractional function $h$, one can build some estimators based on generalized quadratic variations, method used in $[10,7,5]$.

The next section is devoted to the construction of RHMLMs. In Section 3, the regularity of the sample paths of RHMLMs and the asymptotic self-similarity property are studied. The last section deals with the identification of the multifractional function, using localized generalized quadratic variations.

\section{Construction of non-Gaussian multifractional fields}

In this part $M(d \xi)$ is a non vanishing Lévy random measure represented by a Poisson random measure $N(d \xi, d z)$ in the sense of Section 3.12 of [14] but with a control measure that has moments of every order greater than two. $M(d \xi)$ is a Lévy random measure without Brownian component.

Let $N(d \xi, d z)$ be a Poisson random measure on $\mathbb{R}^{d} \times \mathbb{C}$. Here the mean measure $n(d \xi, d z)=\mathbb{E} N(d \xi, d z)=$ $d \xi \nu(d z)$ satisfies:

$$
\forall p \geqslant 2, \quad \int_{\mathbb{C}}|z|^{p} v(d z)<+\infty .
$$

Moreover $v$ is a nonvanishing measure such that $v(\{0\})=0$. 
If $A$ is a Borel set of $\mathbb{R}^{d} \times \mathbb{C}$ then $N(A)$ is a Poisson random variable of intensity $n(A)$. Moreover if the set $I$ is finite and if the sets $A_{i}, i \in I$, are pairwise disjoint, then the random variables $N\left(A_{i}\right), i \in I$, are independent.

Let us note $\widetilde{N}=N-n$ the compensated Poisson measure. It is classical to define the stochastic integral $\int_{\mathbb{R}^{d} \times \mathbb{C}} \varphi(\xi, z) \tilde{N}(d \xi, d z)$ for every function $\varphi: \mathbb{R}^{d} \times \mathbb{C} \rightarrow \mathbb{C}$ such that $\varphi$ is in $L^{2}\left(\mathbb{R}^{d} \times \mathbb{C}\right)$ for the measure $n(d \xi, d z)$. By construction the map $\varphi \mapsto \int \varphi d \tilde{N}$ is an isometry from $L^{2}\left(\mathbb{R}^{d} \times \mathbb{C}\right)$ onto a subset of $L^{2}(\Omega)$ :

$$
\mathbb{E}\left[\left|\int_{\mathbb{R}^{d} \times \mathbb{C}} \varphi(\xi, z) \tilde{N}(d \xi, d z)\right|^{2}\right]=\int_{\mathbb{R}^{d} \times \mathbb{C}}|\varphi(\xi, z)|^{2} n(d \xi, d z),
$$

for every $\varphi \in L^{2}\left(\mathbb{R}^{d} \times \mathbb{C}\right)$.

Notice that if $\varphi$ is real so is $\int \varphi d \tilde{N}$. Let us denote by $\Re(z)$ the real part of a complex $z$ and by $\Im(z)$ its imaginary part.

The law of the random variable $\int \varphi d \tilde{N}$ is given by its characteristic function

$$
\mathbb{E}\left[\exp \left(i\left(u \int \Re(\varphi) d \tilde{N}+v \int \Im(\varphi) d \tilde{N}\right)\right)\right]
$$

which is equal to

$$
\exp \left[\int_{\mathbb{R}^{d} \times \mathbb{C}}[\exp (i(u \Re(\varphi)+v \Im(\varphi)))-1-i(u \Re(\varphi)+v \Im(\varphi))] d \xi v(d z)\right],
$$

where $(u, v) \in \mathbb{R}^{2}$. Let us remark that $\int \varphi d \tilde{N}$ is a centered random variable.

Following [6],

$$
\int_{\mathbb{R}^{d}} f(\xi) M(d \xi) \stackrel{\text { def }}{=} \int_{\mathbb{R}^{d} \times \mathbb{C}}[f(\xi) z+f(-\xi) \bar{z}] \tilde{N}(d \xi, d z)
$$

where $f \in L^{2}\left(\mathbb{R}^{d}\right)$.

Then if

$$
\forall \xi \in \mathbb{R}^{d}, \quad f(-\xi)=\overline{f(\xi)}
$$

$\int_{\mathbb{R}^{d}} f(\xi) M(d \xi)$ is a real centered random variable.

As in [6], the control measure $v(d z)$ is assumed to be rotationally invariant. Let $P$ be the map $P\left(\rho \mathrm{e}^{i \theta}\right)=$ $(\theta, \rho) \in[0,2 \pi) \times \mathbb{R}_{*}^{+}$. The measure $v(d z)$ satisfies the following property:

$$
P(v(d z))=d \theta v_{\rho}(d \rho)
$$

where $d \theta$ is the uniform measure on $[0,2 \pi)$.

Then, when $f$ satisfies (3),

$$
\mathbb{E}\left[\left|\int_{\mathbb{R}^{d}} f(\xi) M(d \xi)\right|^{2}\right]=4 \pi\|f\|_{L^{2}\left(\mathbb{R}^{d}\right)}^{2} \int_{0}^{+\infty} \rho^{2} v_{\rho}(d \rho) .
$$

A real harmonizable multifractional Lévy motion $X_{h}$ will be characterized by a locally $\beta$-Hölder function $h$. On the other hand, we are interested in the pointwise Hölder exponent of $X_{h}$ at point $x$. Therefore let us precise the definition of these two notions.

Definition 2.1. Let $\beta>0$. Let $f: \mathbb{R}^{d} \rightarrow \mathbb{R}$ be a function on $\mathbb{R}^{d}$.

1. $f$ is a $\beta$-Hölder function on $U \subset \mathbb{R}^{d}$ if

$$
\exists C \in \mathbb{R}^{+}, \forall(x, y) \in U^{2}, \quad|f(x)-f(y)| \leqslant C\|x-y\|^{\beta} .
$$


2. $f$ is a locally $\beta$-Hölder function on $\mathbb{R}^{d}$ if for every compact set $K \subset \mathbb{R}^{d}, f$ is a $\beta$-Hölder function on $K$.

3. Let $x \in \mathbb{R}^{d}$. Then

$$
\alpha_{f}(x)=\sup \left\{\alpha>0, \lim _{y \rightarrow 0} \frac{f(x+y)-f(x)}{\|y\|^{\alpha}}=0\right\}
$$

is called the pointwise Hölder exponent of the function $f$ at point $x$.

Let us remark that if $f$ is a locally $\beta$-Hölder function on $\mathbb{R}^{d}$ then for every $x \in \mathbb{R}^{d}, \alpha_{f}(x) \geqslant \beta$.

For every $U \subset \mathbb{R}^{d}$ and every $\beta>0$, let

$$
\|f\|_{\beta, U}=\sup _{\substack{(u, v) \in K^{2} \\ u \neq v}} \frac{|f(u)-f(v)|}{\|u-v\|^{\beta}} .
$$

In particular, $f$ is a locally $\beta$-Hölder function if and only if for every compact set $K \subset \mathbb{R}^{d},\|f\|_{\beta, K}<+\infty$.

Let us now introduce the class of real harmonizable multifractional Lévy motions.

Definition 2.2. Let $\beta>0$ and let $\left.h: \mathbb{R}^{d} \rightarrow\right] 0,1\left[\right.$ be a locally $\beta$-Hölder function on $\mathbb{R}^{d}$. We suppose that the Lévy random measure $M(d \xi)$ satisfies the finite moment assumption (2) and the rotational invariance (4). Let $(a, b) \in \mathbb{R}^{2}$. A real harmonizable multifractional Lévy motion (in short RHMLM) is a real valued field which admits a harmonizable representation

$$
X_{h}(x)=\int_{\mathbb{R}^{d}} \frac{\mathrm{e}^{-i x \cdot \xi}-1}{\|\xi\|^{d / 2+h(x)}} L(d \xi),
$$

where $L(d \xi)=a M(d \xi)+b W(d \xi)$ is the sum of the Lévy random measure $a M(d \xi)$ and of an independent Wiener measure $b W(d \xi)$.

Consequently, $X_{h}$ is the sum of two independent fields, one of which is a multifractional Brownian motion. In particular the MBM is the RHMLM obtained for $L(d \xi)=W(d \xi)$. Assuming (4), RHFLMs have stationary increments. It does not remain true for RHMLMs.

In all the sequel, $\left.h: \mathbb{R}^{d} \rightarrow\right] 0,1\left[\right.$ is a locally $\beta$-Hölder function on $\mathbb{R}^{d}$ and $X_{h}$ is the RHMLM associated with $h$.

\section{Some properties of real harmonizable multifractional Lévy motions}

This section deals with two properties that RHMLMs share with the MBM. The RHMLM $X_{h}$ has locally $H$ Hölder sample paths at point $x$ for every $H<\min (h(x), \beta)$. Moreover, if for every $x, 0<h(x)<\beta$, then $X_{h}$ is lass with tangent FBM at point $x$. From this last property, one deduces that the Hölder exponent of $X_{h}$ at point $x$ is almost surely equal to $h(x)$.

As these properties have already be shown in [8] in the case of the MBM, i.e. in the case where $L(d \xi)=W(d \xi)$, we suppose for sake of simplicity that $L(d \xi)=M(d \xi)$.

\subsection{Preliminary lemmas}

Usually, to get the regularity of the trajectories and the lass property, one estimates

$$
\mathbb{E}\left[\left|X_{h}(x)-X_{h}(y)\right|^{q}\right], \quad q \in \mathbb{N}^{*},
$$


where $q \in \mathbb{N}^{*}=\mathbb{N} \backslash\{0\}$. Nevertheless when $h(x)>1-d / 2$ and $q \geqslant 3, X_{h}(x)$ may have an infinite moment of order $q$. Thus the field $X_{h}$ is split into two fields $X_{h}=X_{h}^{+}+X_{h}^{-}$where $X_{h}^{+}$has moments of every order and $X_{h}^{-}$ has almost surely locally $\beta$-Hölder sample paths. Then one can estimate

$$
\mathbb{E}\left[\left|X_{h}^{+}(x)-X_{h}^{+}(y)\right|^{q}\right], \quad q \in \mathbb{N}^{*} .
$$

Let $n \in \mathbb{N}$ and

$$
P_{n}(t)=\sum_{k=1}^{n} \frac{t^{k}}{k !}, \quad \text { with convention } P_{0}(t)=0 .
$$

Then

$$
g_{n}^{+}(x, \xi)=\frac{\mathrm{e}^{-i x \cdot \xi}-1-P_{n}(-i x \cdot \xi) \mathbf{1}_{\|\xi\| \leqslant 1}}{\|\xi\|^{d / 2+h(x)}}
$$

and

$$
g_{n}^{-}(x, \xi)=\frac{P_{n}(-i x \cdot \xi) \mathbf{1}_{\|\xi\| \leqslant 1}}{\|\xi\|^{d / 2+h(x)}}
$$

are in $L^{2}\left(\mathbb{R}^{d}\right)$ for every $x \in \mathbb{R}^{d}$.

Therefore $X_{h}=X_{h, n}^{+}+X_{h, n}^{-}$with

$$
\begin{aligned}
& X_{h, n}^{+}(x)=\int_{\mathbb{R}^{d}} g_{n}^{+}(x, \xi) L(d \xi) \\
& \text { and } X_{h, n}^{-}(x)=\int_{\mathbb{R}^{d}} g_{n}^{-}(x, \xi) L(d \xi) .
\end{aligned}
$$

Notice that

$$
X_{h}=X_{h, 0}^{+} .
$$

Moreover $g_{n}^{+}(x, \cdot) \in L^{q}\left(\mathbb{R}^{d}\right)$ for every $q \geqslant 2$ such that

$$
(n+1-d / 2-h(x)) q>-d .
$$

Consequently when $n \geqslant d / 2, g_{n}^{+}(x, \cdot) \in L^{q}\left(\mathbb{R}^{d}\right)$ for every $q \geqslant 2$ and every $x \in \mathbb{R}^{d}$. In this case $X_{h, n}^{+}$has moments of every order (see next proposition given in [6]).

Proposition 3.1. Let $p \in \mathbb{N}^{*}, f \in L^{2}\left(\mathbb{R}^{d}\right) \cap L^{2 p}\left(\mathbb{R}^{d}\right)$ and suppose that $f$ satisfies (3) then $\int_{\mathbb{R}^{d}} f(\xi) M(d \xi)$ is in $L^{2 p}(\Omega)$ and

$$
\mathbb{E}\left[\left(\int_{\mathbb{R}^{d}} f(\xi) M(d \xi)\right)^{2 p}\right]=\sum_{m=1}^{p}(2 \pi)^{m} \sum_{L_{m}} \prod_{q=1}^{m} \frac{\left(2 l_{q}\right) !\|f\|_{2 l_{q}}^{2 l_{q}} \int_{0}^{+\infty} \rho^{2 l_{q}} v_{\rho}(d \rho)}{\left(l_{q} !\right)^{2}}
$$

where $\sum_{L_{m}}$ stands for the sum over the set of partitions $L_{m}$ of $\{1, \ldots, 2 p\}$ in $m$ subsets $K_{q}$ such that the cardinality of $K_{q}$ is $2 l_{q}$ with $l_{q} \geqslant 1$ and where $\|f\|_{2 l_{q}}$ is the $L^{2 l_{q}}\left(\mathbb{R}^{d}\right)$ norm of $f$.

Using this proposition, one can compute

$$
\mathbb{E}\left[\left(X_{h, n}^{+}(x)-X_{h, n}^{+}(y)\right)^{2 p}\right]
$$


with the help of some $L^{2 q}$-norms of the deterministic map $\xi \mapsto g_{n}(x, y, \xi)$, where

$$
g_{n}(x, y, \xi)=g_{n}^{+}(x, \xi)-g_{n}^{+}(y, \xi) .
$$

To estimate these norms, $g_{n}$ is split into $g_{n}=g_{n, 1}+g_{n, 2}$ with

$$
g_{n, 1}(x, y, \xi)=\frac{\mathrm{e}^{-i x \cdot \xi}-\mathrm{e}^{-i y \cdot \xi}+\left[P_{n}(-i y \cdot \xi)-P_{n}(-i x \cdot \xi)\right] \mathbf{1}_{\|\xi\| \leqslant 1}}{\|\xi\|^{d / 2+h(y)}}
$$

and

$$
g_{n, 2}(x, y, \xi)=\frac{\mathrm{e}^{-i x \cdot \xi}-1-P_{n}(-i x \cdot \xi) \mathbf{1}_{\|\xi\| \leqslant 1}}{\|\xi\|^{d / 2}}\left[\frac{1}{\|\xi\|^{h(x)}}-\frac{1}{\|\xi\|^{h(y)}}\right] .
$$

If $X_{h}$ is a RHFLM of index $H$, i.e. if $h$ is equal to a constant $H$, notice that $g_{n, 2}=0$. One of the main difference between the studies of RHFLMs and RHMLMs lies in the study of the properties of $g_{n, 2}$.

Lemma 3.2. Let $K \subset \mathbb{R}^{d}$ be a compact set. Suppose that $q \geqslant 2$ is such that $q(n-d / 2) \geqslant-d$. Then there exists a nonnegative constant $C=C(K, q)$ such that

$$
\forall(x, y) \in K^{2}, \quad\left\|g_{n, 1}(x, y, \cdot)\right\|_{q}^{q} \leqslant C\|x-y\|^{q h(y)} .
$$

Proof. Let $(x, y) \in K^{2}$ and let us note $I_{1}=\left\|g_{n, 1}(x, y, \cdot)\right\|_{q}^{q}$.

$$
I_{1}(x, y)=I_{11}(x, y)+I_{12}(x, y)
$$

where

$$
\begin{aligned}
& I_{11}(x, y)=\int_{\|\xi\| \leqslant 1} \frac{\left|\mathrm{e}^{-i x \cdot \xi}-\mathrm{e}^{-i y \cdot \xi}+P_{n}(-i y \cdot \xi)-P_{n}(-i x \cdot \xi)\right|^{q}}{\|\xi\|^{q d / 2+q h(y)}} d \xi \text { and } \\
& I_{12}(x, y)=\int_{\|\xi\| \geqslant 1} \frac{\left|\mathrm{e}^{-i x \cdot \xi}-\mathrm{e}^{-i y \cdot \xi}\right|^{q}}{\|\xi\|^{q d / 2+q h(y)}} d \xi .
\end{aligned}
$$

By Taylor expansion,

$$
\left|\mathrm{e}^{-i x \cdot \xi}-\mathrm{e}^{-i y \cdot \xi}+P_{n}(-i y \cdot \xi)-P_{n}(-i x \cdot \xi)\right| \leqslant C\|x-y\|\|\xi\|^{n+1} .
$$

Let us define $M_{K}=\max _{K} h$. If $0<\|\xi\| \leqslant 1$, then

$$
\frac{1}{\|\xi\|^{h(y)}} \leqslant \frac{1}{\|\xi\|^{M_{K}}} \text {. }
$$

Consequently,

$$
I_{11}(x, y) \leqslant C\|x-y\|^{q} \int_{\|\xi\| \leqslant 1} \frac{1}{\|\xi\|^{q d / 2+q\left(M_{K}-n-1\right)}} d \xi .
$$

This last integral is defined since $q(n-d / 2) \geqslant-d$ and so

$$
I_{11}(x, y) \leqslant C\|x-y\|^{q} .
$$

It remains to study $I_{12}$. Unfortunately Taylor expansion gives an infinite bound. Let us suppose that $x \neq y$. One splits $I_{12}(x, y)$ into the integrals

$$
J_{1}(x, y)=\int_{\substack{\|x-y\|\|\xi\| \geqslant 1 \\\|\xi\| \geqslant 1}} \frac{\left|\mathrm{e}^{-i(x-y) \cdot \xi}-1\right|^{q}}{\|\xi\|^{q d / 2+q h(y)}} d \xi \quad \text { and } \quad J_{2}(x, y)=\int_{1 \leqslant\|\xi\| \leqslant \frac{1}{\|x-y\|}} \frac{\left|\mathrm{e}^{-i(x-y) \cdot \xi}-1\right|^{q}}{\|\xi\|^{q d / 2+q h(y)}} d \xi .
$$


Then one can easily see that

$$
J_{1}(x, y) \leqslant C\|x-y\|^{d(q / 2-1)+q h(y)} .
$$

By Taylor expansion,

$$
J_{2}(x, y) \leqslant C\|x-y\|^{q} \int_{1}^{\frac{1}{\pi x-y \|}} \rho^{d-1-q d / 2-q h(y)+q} d \rho .
$$

Then

$$
J_{2}(x, y) \leqslant C\|x-y\|^{q\left(1+h(y)-M_{K}\right)} \int_{1}^{\frac{1}{\pi x-y \|}} \rho^{d-1-q d / 2-q M_{K}+q} d \rho .
$$

Consequently, by evaluating the last integral, one obtains that

$$
J_{2}(x, y) \leqslant C\|x-y\|^{q h(y)} .
$$

Let us now study $g_{n, 2}$.

Lemma 3.3. Let $K \subset \mathbb{R}^{d}$ be a compact set. Let us note $m_{K}=\min _{K} h$ and $M_{K}=\max _{K} h$. Suppose that $q \geqslant 2$ is such that $q(n-d / 2) \geqslant-d$. Then

$$
\forall(x, y) \in K^{2}, \quad\left\|g_{n, 2}(x, y, \cdot)\right\|_{q}^{q} \leqslant C\|x-y\|^{q \beta},
$$

where

$$
C=\|h\|_{\beta, K}^{q} \sup _{u \in K} J(u) \in \mathbb{R}^{+}
$$

with

$$
J(u)=\int_{\mathbb{R}^{d}} \frac{\left|\mathrm{e}^{-i u \cdot \xi}-1-P_{n}(-i u \cdot \xi) \mathbf{1}_{\|\xi\| \leqslant 1}\right|^{q}|\ln \|\xi\||^{q}}{\|\xi\|^{d q / 2}}\left[\frac{1}{\|\xi\|^{m_{K}}}+\frac{1}{\|\xi\|^{M_{K}}}\right]^{q} d \xi .
$$

Proof. Let $(x, y) \in K^{2}$ and let us note $I_{2}=\left\|g_{n, 2}(x, y, \cdot)\right\|_{q}^{q}$. By the Mean Value Theorem,

$$
\frac{1}{\|\xi\|^{h(x)}}-\frac{1}{\|\xi\|^{h(y)}}=\frac{-(h(x)-h(y)) \ln \|\xi\|}{\|\xi\|^{c \xi, x, y}}
$$

where $c_{\xi, x, y}$ is between $h(x)$ and $h(y)$. Therefore $m_{K} \leqslant c_{\xi, x, y} \leqslant M_{K}$.

Furthermore

$$
\frac{1}{\|\xi\|^{c \xi, x, y}} \leqslant \frac{1}{\|\xi\|^{m_{K}}} \mathbf{1}_{\|\xi\| \geqslant 1}+\frac{1}{\|\xi\|^{M_{K}}} \mathbf{1}_{\|\xi\|<1} .
$$

Then

$$
\frac{1}{\|\xi\|^{c_{\xi, x, y}}} \leqslant \frac{1}{\|\xi\|^{m_{K}}}+\frac{1}{\|\xi\|^{M_{K}}}
$$

Consequently,

$$
\left|\frac{1}{\|\xi\|^{h(x)}}-\frac{1}{\|\xi\|^{h(y)}}\right| \leqslant|h(x)-h(y)||\ln \|\xi\||\left[\frac{1}{\|\xi\|^{m_{K}}}+\frac{1}{\|\xi\|^{M_{K}}}\right] .
$$


Therefore $I_{2}(x, y) \leqslant|h(x)-h(y)|^{q} J(x)$, where $J(x)$ is equal to

$$
\int_{\mathbb{R}^{d}} \frac{\left|\mathrm{e}^{-i x \cdot \xi}-1-P_{n}(-i x \cdot \xi) \mathbf{1}_{\|\xi\| \leqslant 1}\right|^{q}|\ln \|\xi\||^{q}}{\|\xi\|^{d q / 2}}\left[\frac{1}{\|\xi\|^{m_{K}}}+\frac{1}{\|\xi\|^{M_{K}}}\right]^{q} d \xi .
$$

It is straightforward to prove that $\sup _{u \in K} J(u)<+\infty$. Moreover since $h$ is a locally $\beta$-Hölder function on $\mathbb{R}^{d}$,

$$
|h(x)-h(y)| \leqslant\|h\|_{\beta, K}\|x-y\|^{\beta}
$$

and then

$$
I_{2}(x, y) \leqslant\|h\|_{\beta, K}^{q} \sup _{u \in K} J(u)\|x-y\|^{\beta q} .
$$

Since $g_{n}=g_{n, 1}+g_{n, 2}$, by applying Minkowski inequality, Proposition 3.1 and Lemmas 3.2 and 3.3, one can prove:

Lemma 3.4. Let $K \subset \mathbb{R}^{d}$ be a compact set and $n \geqslant d / 2$, then for every $p \in \mathbb{N}^{*}$, there exists a nonnegative constant $C=C(K, p)$ such that

$$
\forall(x, y) \in K^{2}, \quad \mathbb{E}\left[\left(X_{h, n}^{+}(x)-X_{h, n}^{+}(y)\right)^{2 p}\right] \leqslant C\|y-x\|^{2 p m},
$$

where $m=\min (h(y), \beta)$.

Let us notice that by symmetry, $m$ can be replaced by

$$
m^{\prime}=\min (\max (h(x), h(y)), \beta) \text {. }
$$

It remains now to study $X_{h, n}^{-}$. Please note that as usual the regularity of the trajectories is given for a modification $\widetilde{X}^{-}$of $X_{h, n}^{-}$, i.e. for a field $\widetilde{X}^{-}$such that

$$
\forall x \in \mathbb{R}^{d}, \quad \mathbb{P}\left(\tilde{X}^{-}(x)=X_{h, n}^{-}(x)\right)=1 .
$$

Lemma 3.5. There exists a modification of the field $X_{h, n}^{-}$that has, with probability one, locally $\beta$-Hölder sample paths.

Remark. When $h$ is $\mathcal{C}^{1}$, there exists a modification of the field $X_{h, n}^{-}$such that with probability one, $X_{h, n}^{-} \in \mathcal{C}^{1}$.

Proof. Notice that for every $x \in \mathbb{R}^{d}$

$$
X_{h, n}^{-}(x)=Z_{n}(x, h(x)) \text {, }
$$

where the field $\left(Z_{n}(x, y)\right)_{\left.(x, y) \in \mathbb{R}^{d} \times\right] 0,1[}$ is defined as follows:

$$
Z_{n}(x, y)=\int_{\|\xi\| \leqslant 1} \frac{P_{n}(-i x \cdot \xi)}{\|\xi\|^{d / 2+y}} L(d \xi) .
$$

Then since $h$ is a locally $\beta$-Hölder map with values in $] 0,1[$, it is sufficient to prove that there exists a modification of the field $Z_{n}$ such that $\mathbb{P}\left(Z_{n} \in \mathcal{C}^{1}\right)=1$.

Let us define

$$
Y_{\alpha}(y)=\int_{\|\xi\| \leqslant 1} \frac{i^{|\alpha|} \prod_{j=1}^{d} \xi_{j}^{\alpha_{j}}}{\|\xi\|^{d / 2+y}} L(d \xi)
$$


where $\alpha \in \mathbb{N}^{d}$ is such that $1 \leqslant|\alpha|=\sum_{j=1}^{n} \alpha_{j} \leqslant n$ and $\left.y \in\right] 0,1\left[\right.$. One shows that for every $\alpha$, the field $Y_{\alpha}$ admits a modification which has almost surely $\mathcal{C}^{1}$-sample paths on ]0,1[. Then since $P_{n}$ is a polynomial, the same holds for $Z_{n}$.

Let $\alpha \in \mathbb{N}^{d}$ such that $1 \leqslant \sum_{j=1}^{n} \alpha_{j} \leqslant n$ and $\left.\eta \in\right] 0,1[$. One can prove with Taylor expansion the existence of a constant $C>0$ such that

1. $\mathbb{E}\left[\left|Y_{\alpha}(y+\delta)-Y_{\alpha}(y)\right|^{2}\right] \leqslant C|\delta|^{2}$, for every $y \in[\eta, 1-\eta]$ and $\delta$ such that $y+\delta \in[\eta, 1-\eta]$,

2. $\mathbb{E}\left[\left|Y_{\alpha}(y+\delta)+Y_{\alpha}(y-\delta)-2 Y_{\alpha}(y)\right|^{2}\right] \leqslant C|\delta|^{4}$, for every $y \in[\eta, 1-\eta]$ and $\delta$ such that $(y+\delta, y-\delta) \in$ $[\eta, 1-\eta]^{2}$.

According to [9], see p. 69, these statements imply the existence of a modification of $Y_{\alpha}$ which has almost surely $\mathcal{C}^{1}$-sample paths. And so the same holds for $Z_{n}$.

\subsection{Trajectories regularity}

There exist modifications of the MBM whose sample paths are locally Hölder. Here an analogous result in the case of RHMLMs is shown.

Proposition 3.6. Let $K \subset \mathbb{R}^{d}$ be a compact set.

Then for every $H<\min \left(\min _{K} h, \beta\right)$, there exists a modification of the RHMLM $X_{h}$ which has $H$-Hölder sample paths on $K$.

Proof. Let $H<\min \left(\min _{K} h, \beta\right)$ and $n \geqslant d / 2$.

As a consequence of Lemma 3.4 and of Kolmogorov Theorem, there exists a modification of the field $X_{h, n}^{+}$ whose sample paths are $H$-Hölder on $K$. Then since $X_{h, n}^{-}$has almost surely locally $\beta$-Hölder sample paths (see Lemma 3.5) and $X_{h}=X_{h, n}^{+}+X_{h, n}^{-}$, the proof is done.

Then Proposition 3.6 give us a lower bound for the pointwise Hölder exponent $\alpha_{X_{h}}(x)$ of $X_{h}$ at point $x$ :

$$
\alpha_{X_{h}}(x) \geqslant \min (h(x), \beta) .
$$

\subsection{Asymptotic self-similarity}

Proposition 3.7. Suppose that for every $x \in \mathbb{R}^{d}, h(x)<\beta$. Then the real harmonizable multifractional Lévy motion $X_{h}$ is locally self-similar with multifractional function $h$ in the sense that for every fixed $x \in \mathbb{R}^{d}$ :

$$
\lim _{\varepsilon \rightarrow 0^{+}}\left(\frac{X_{h}(x+\varepsilon u)-X_{h}(x)}{\varepsilon^{h(x)}}\right)_{u \in \mathbb{R}^{d}} \stackrel{(d)}{=} C_{x}\left(B_{h(x)}(u)\right)_{u \in \mathbb{R}^{d}},
$$

where the convergence is a convergence in distribution on the space of continuous functions endowed with the topology of the uniform convergence on compact sets, $B_{h(x)}$ is a standard FBM of index $h(x)$ and

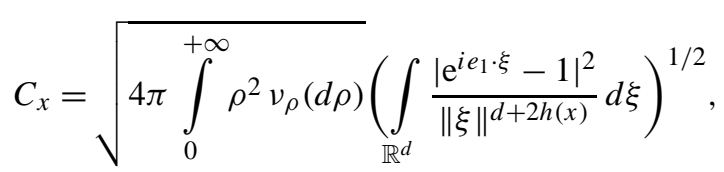

with $e_{1}=(1,0, \ldots, 0) \in \mathbb{R}^{d}$. 
Remark. This proposition is stated in the case where $L(d \xi)=M(d \xi)$. However it remains true when $L(d \xi)=$ $a M(d \xi)+b W(d \xi)$ is as in Definition 2.2, but with

$$
C_{x}=\left[4 a^{2} \pi \int_{0}^{+\infty} \rho^{2} v_{\rho}(d \rho)+b^{2}\right]^{1 / 2}\left[\int_{\mathbb{R}^{d}} \frac{\left|\mathrm{e}^{i e_{1} \cdot \xi}-1\right|^{2}}{\|\xi\|^{d+2 h(x)}} d \xi\right]^{1 / 2} .
$$

Proof. We first prove the convergence of the finite dimensional margins. Next a tightness property is shown. It is a direct consequence of Lemmas 3.4 and 3.5.

Let us recall that $X_{h}=X_{h, 0}^{+}$and that

$$
g_{0}(u, v, \xi)=g_{0}^{+}(u, \xi)-g_{0}^{+}(v, \xi)=\frac{\mathrm{e}^{-i u \cdot \xi}-1}{\|\xi\|^{d / 2+h(u)}}-\frac{\mathrm{e}^{-i v \cdot \xi}-1}{\|\xi\|^{d / 2+h(v)}} .
$$

Let us fixed $x \in \mathbb{R}^{d}$. Then for every $\varepsilon>0$ and $u \in \mathbb{R}^{d}$,

$$
Y_{\varepsilon}(u)=\frac{X_{h}(x+\varepsilon u)-X_{h}(x)}{\varepsilon^{h(x)}}=\frac{1}{\varepsilon^{h(x)}} \int_{\mathbb{R}^{d}} g_{0}(x+\varepsilon u, x, \xi) L(d \xi) .
$$

In the sequel we shall need to use the decomposition of $g_{0}$ into $g_{0}=g_{1}+g_{2}$ where $g_{1}=g_{0,1}$ is defined by (10) and $g_{2}=g_{0,2}$ by (11).

Convergence of the finite dimensional margins. Let $p \in \mathbb{N}^{*}, u=\left(u_{1}, \ldots, u_{p}\right) \in\left(\mathbb{R}^{d}\right)^{p}, v=\left(v_{1}, \ldots, v_{p}\right) \in \mathbb{R}^{p}$ and

$$
g_{j}^{\varepsilon}(\xi, z)=\frac{2}{\varepsilon^{h(x)}} \Re\left(z \sum_{k=1}^{p} v_{k} g_{j}\left(x+\varepsilon u_{k}, x, \xi\right)\right), \quad j=0,1,2 .
$$

Then since $\sum_{k=1}^{p} v_{k} Y_{\varepsilon}\left(u_{k}\right)=\int_{\mathbb{R}^{d}} g_{0}^{\varepsilon}(\xi, z) \tilde{N}(d \xi, d z)$,

$$
\mathbb{E}\left[\exp \left(i \sum_{k=1}^{p} v_{k} Y_{\varepsilon}\left(u_{k}\right)\right)\right]=\exp \left(\psi_{\varepsilon}(u, v)\right)
$$

with $\psi_{\varepsilon}(u, v)=\int_{\mathbb{R}^{d} \times \mathbb{C}}\left[\exp \left(i g_{0}^{\varepsilon}(\xi, z)\right)-1-i g_{0}^{\varepsilon}(\xi, z)\right] d \xi \nu(d z)$.

Then $\psi_{\varepsilon}(u, v)=I_{1}^{\varepsilon}+I_{2}^{\varepsilon}+I_{3}^{\varepsilon}$, where

$$
\begin{aligned}
I_{1}^{\varepsilon} & =\int_{\mathbb{R}^{d} \times \mathbb{C}}\left[\exp \left(i g_{1}^{\varepsilon}(\xi, z)\right)-1-i g_{1}^{\varepsilon}(\xi, z)\right] d \xi v(d z), \\
I_{2}^{\varepsilon} & =\int_{\mathbb{R}^{d} \times \mathbb{C}}\left[\exp \left(i g_{2}^{\varepsilon}(\xi, z)\right)-1-i g_{2}^{\varepsilon}(\xi, z)\right] d \xi v(d z), \\
I_{3}^{\varepsilon} & =\int_{\mathbb{R}^{d} \times \mathbb{C}}\left[\exp \left(i g_{0}^{\varepsilon}(\xi, z)\right)+1-\exp \left(i g_{1}^{\varepsilon}(\xi, z)\right)-\exp \left(i g_{2}^{\varepsilon}(\xi, z)\right)\right] d \xi v(d z) .
\end{aligned}
$$

Since $g_{j}^{\varepsilon} \in L^{2}\left(\mathbb{R}^{d} \times \mathbb{C}\right)$, these three integrals are defined.

1. Study of $I_{1}^{\varepsilon}$. By rotational invariance of the measure $v(d z)$ and by applying the change of variable $\lambda=\varepsilon \xi$, one obtains

$$
I_{1}^{\varepsilon}=\int_{\mathbb{R}^{d} \times \mathbb{C}} l^{\varepsilon}(\lambda, z) d \lambda v(d z)
$$


where $l^{\varepsilon}(\lambda, z)=\frac{1}{\varepsilon^{d}}\left[\exp \left(\varepsilon^{d / 2} \tilde{g}_{1}(\lambda, z)\right)-1-\varepsilon^{d / 2} \tilde{g}_{1}(\lambda, z)\right]$ with

$$
\tilde{g}_{1}(\lambda, z)=2 i \Re\left(z \sum_{k=1}^{p} v_{k} \frac{\mathrm{e}^{-i u_{k} \cdot \lambda}-1}{\|\lambda\|^{d / 2+h(x)}}\right) .
$$

Then as $\varepsilon \rightarrow 0_{+}$a dominated convergence argument yields that

$$
\lim _{\varepsilon \rightarrow 0+} I_{1}^{\varepsilon}=-2 \pi \int_{0}^{+\infty} \rho^{2} v_{\rho}(d \rho) \int_{\mathbb{R}^{d}}\left[\sum_{k=1}^{p} v_{k} \frac{\left|\mathrm{e}^{-i u_{k} \cdot \lambda}-1\right|}{|\lambda|^{d / 2+h(x)}}\right]^{2} d \lambda .
$$

2. Study of $I_{2}^{\varepsilon}$. Since for every $t \in \mathbb{R},\left|\mathrm{e}^{i t}-1-i t\right| \leqslant|t|^{2} / 2$,

$$
\left|I_{2}^{\varepsilon}\right| \leqslant \frac{1}{2} \int_{\mathbb{R}^{d} \times \mathbb{C}}\left|g_{2}^{\varepsilon}(\xi, z)\right|^{2} d \xi v(d z), \quad \text { i.e. } \quad\left|I_{2}^{\varepsilon}\right| \leqslant \frac{C}{\varepsilon^{2 h(x)}}\left\|\sum_{k=1}^{p} v_{k} g_{2}\left(x+\varepsilon u_{k}, x, \cdot\right)\right\|_{2}^{2} .
$$

One applies Lemma 3.3 and obtains that for $\varepsilon \leqslant 1$ and for every $k$,

$$
\left\|g_{2}\left(x+\varepsilon u_{k}, x, \cdot\right)\right\|_{2}^{2} \leqslant C \varepsilon^{2 \beta} .
$$

Then as $\beta>h(x), \lim _{\varepsilon \rightarrow 0_{+}}\left\|g_{2}\left(x+\varepsilon u_{k}, x, \cdot\right)\right\|_{2} / \varepsilon^{h(x)}=0$. And so by applying Minkowski inequality,

$$
\lim _{\varepsilon \rightarrow 0_{+}} I_{2}^{\varepsilon}=0 .
$$

3. Study of $I_{3}^{\varepsilon}$. One can notice that

$$
\left|I_{3}^{\varepsilon}\right| \leqslant \int_{\mathbb{R}^{d} \times \mathbb{C}}\left|g_{1}^{\varepsilon}(\xi, z)\right|\left|g_{2}^{\varepsilon}(\xi, z)\right| d \xi \nu(d z) .
$$

Because of Lemma 3.2, for $\varepsilon \leqslant 1$

$$
\int_{\mathbb{R}^{d}}\left|g_{1}^{\varepsilon}(\xi, z)\right|^{2} d \xi v(d z) \leqslant C .
$$

Then Cauchy-Schwarz inequality implies that

$$
\lim _{\varepsilon \rightarrow 0_{+}} I_{3}^{\varepsilon}=0 .
$$

Consequently, $\lim _{\varepsilon \rightarrow 0^{+}} \mathbb{E}\left[\exp \left(i \sum_{k=1}^{p} v_{k} Y_{\varepsilon}\left(u_{k}\right)\right)\right]$ is equal to

$$
\exp \left(-2 \pi \int_{0}^{+\infty} \rho^{2} v_{\rho}(d \rho) \int_{\mathbb{R}^{d}}\left[\sum_{k=1}^{p} v_{k} \frac{\left|\mathrm{e}^{-i u_{k} \cdot \lambda}-1\right|}{|\lambda|^{d / 2+h(x)}}\right]^{2} d \lambda\right),
$$

and then

$$
\lim _{\varepsilon \rightarrow 0^{+}} \mathbb{E}\left[\exp \left(i \sum_{k=1}^{p} v_{k} Y_{\varepsilon}\left(u_{k}\right)\right)\right]=\exp \left(-\frac{C_{x}^{2}}{2} \operatorname{Var} \sum_{k=1}^{p} v_{k} B_{h(x)}\left(u_{k}\right)\right),
$$

where $B_{h(x)}$ is a standard fractional Brownian motion of index $h(x)$ and $C_{x}$ is defined in the statement of the proposition, which concludes the proof of the convergence of the finite margins. 
Tightness. Let $n \geqslant d / 2$,

$$
Y_{\varepsilon}^{+}(u)=\frac{X_{h, n}^{+}(x+\varepsilon u)-X_{h, n}^{+}(x)}{\varepsilon^{h(x)}} \quad \text { and } \quad Y_{\varepsilon}^{-}(u)=\frac{X_{h, n}^{-}(x+\varepsilon u)-X_{h, n}^{-}(x)}{\varepsilon^{h(x)}} .
$$

Then $Y_{\varepsilon}=Y_{\varepsilon}^{+}+Y_{\varepsilon}^{-}$.

Since $X_{h, n}^{-}$has locally $\beta$-Hölder sample paths (see Lemma 3.5) and since $h(x)<\beta$, it is clear that

$$
\lim _{\varepsilon \rightarrow 0_{+}}\left(\frac{X_{h, n}^{-}(x+\varepsilon u)-X_{h, n}^{-}(x)}{\varepsilon^{h(x)}}\right)_{u \in \mathbb{R}^{d}} \stackrel{(d)}{=} 0 .
$$

Consequently, $\left(Y_{\varepsilon}^{-}\right)_{\varepsilon>0}$ is a tight family. Let us now prove the tightness of $\left(Y_{\varepsilon}^{+}\right)_{\varepsilon}$. Notice that $Y_{\varepsilon}^{+}(0)=0$ and so that $\left(Y_{\varepsilon}^{+}(0)\right)_{\varepsilon}$ is tight.

Let $K \subset \mathbb{R}^{d}$ be a compact set and $r_{0}>0$ such that when $\varepsilon \leqslant 1, x+\varepsilon K \subset K_{0}$ where $K_{0}=\overline{B\left(x, r_{0}\right)}$. Lemma 3.4 is applied to the compact set $K_{0}$. Then for every $p \in \mathbb{N}^{*}$, there exists a constant $C=C\left(p, K_{0}, x\right)>0$ such that for every $\varepsilon \in] 0,1]$ and every $(u, v) \in K^{2}$,

$$
\mathbb{E}\left[\left(Y_{\varepsilon}^{+}(u)-Y_{\varepsilon}^{+}(v)\right)^{2 p}\right] \leqslant C \varepsilon^{2 p(h(x+\varepsilon v)-h(x))}\|u-v\|^{2 p h(x+\varepsilon v)} .
$$

Moreover as $h$ is a locally $\beta$-Hölder map,

$$
\forall \varepsilon \in] 0,1], \forall v \in K, \quad \varepsilon^{2 p(h(x+\varepsilon v)-h(x))} \leqslant C .
$$

Therefore for every $p \in \mathbb{N}^{*}$, there exists a constant $C=C(p, K, x)>0$ such that for every $\left.\left.\varepsilon \in\right] 0,1\right]$ and every $(u, v) \in K^{2}$,

$$
\mathbb{E}\left[\left(Y_{\varepsilon}^{+}(u)-Y_{\varepsilon}^{+}(v)\right)^{2 p}\right] \leqslant C\|u-v\|^{2 p \min _{K_{0}} h} .
$$

One can choose $p \in \mathbb{N}^{*}$ such that $2 p \min _{K_{0}} h>d$, which concludes the proof.

Let us fixed $x \in \mathbb{R}^{d}$. Actually, convergence (14) holds as soon as $h(x)<\alpha_{h}(x)$. Then, one can wonder what happens when $h(x)>\alpha_{h}(x)$. In this case, suppose that

$$
l(x)=\lim _{y \rightarrow 0} \frac{h(x+y)-h(x)}{\|y\|^{\alpha_{h}(x)}}
$$

exists and is not equal to 0 . Then

$$
\lim _{\varepsilon \rightarrow 0^{+}}\left(\frac{X_{h}(x+\varepsilon u)-X_{h}(x)}{\varepsilon^{\alpha_{h}(x)}}\right)_{u \in \mathbb{R}^{d}} \stackrel{(d)}{=} l(x) X^{*}(x)\left(\|u\|^{\alpha_{h}(x)}\right)_{u \in \mathbb{R}^{d}},
$$

where the limit is in distribution for all finite margins of the fields and where

$$
X^{*}(x)=\int_{\mathbb{R}^{d}} \frac{\left(\mathrm{e}^{-i x \cdot \xi}-1\right) \ln \|\xi\|}{\|\xi\|^{d / 2+h(x)}} L(d \xi) .
$$

Let us remark that in (15), the field $\left(\|u\|^{\alpha_{h}(x)}\right)_{u \in \mathbb{R}^{d}}$ is deterministic. Then the randomness of the tangent field is only due to the real-valued random variable $X^{*}(x)$ which does not depend on $u$. Let us precise that one proceeds as in the proof of (14), replacing $\varepsilon^{h(x)}$ by $\varepsilon^{\alpha_{h}(x)}$ in $g_{j}^{\varepsilon}$. The preponderant term is then $I_{2}^{\varepsilon}$.

Corollary 3.8. Suppose that for every $x \in \mathbb{R}^{d}, h(x)<\beta$. Then for every $x \in \mathbb{R}^{d}$, the pointwise Hölder exponent of the RHMLM $X_{h}$ at point $x$ is almost surely equal to $h(x)$.

Proof. It is classical, see Proposition 3.3, p. 109 in [6], to deduce from the lass property that the pointwise Hölder exponent $\alpha_{X_{h}}(x)$ of the RHMLM $X_{h}$ at point $x$ satisfies $\alpha_{X_{h}}(x) \leqslant h(x)$.

Then by (13), $\alpha_{X_{h}}(x)=h(x)$. 


\section{Identification}

In this section, $L(d \xi)=M(d \xi)+\sigma W(d \xi)$ is a Lévy random measure which satisfies the assumptions of Definition 2.2. In particular $W$ and $M$ are two independent measures.

Let $\left.h: \mathbb{R}^{d} \rightarrow\right] 0,1\left[\right.$ be a locally $\beta$-Hölder function and $X_{h}$ be the RHMLM associated with $L(d \xi)$ and $h$. Then

$$
X_{h}(x)=\int_{\mathbb{R}^{d}} \frac{\mathrm{e}^{-i x \cdot \xi}-1}{\|\xi\|^{d / 2+h(x)}} L(d \xi) .
$$

Our aim is to identify the multifractional function $h$ from discrete observations of the field $X_{h}$ on $[0,1]^{d}$. The variance $\sigma$ and the control measure $v(d z)$ are unknown. The field is observed at sampling points $\left(\frac{k_{1}}{N}, \ldots, \frac{k_{d}}{N}\right)$, $0 \leqslant k_{j} \leqslant N, 1 \leqslant j \leqslant d$.

One uses localized generalized quadratic variations, method introduced in [5]. For any $x \in] 0,1\left[^{d}\right.$, we define a $(\varepsilon, N)$-neighborhood of $x$ by

$$
\mathcal{V}_{\varepsilon, N}(x)=\left\{\boldsymbol{p} \in \mathbb{Z}^{d}, \max _{j=1 \ldots d}\left|\frac{p_{j}}{N}-x_{j}\right|<\varepsilon\right\} .
$$

Let $\left(a_{\ell}\right)_{\ell=0 \ldots K}$ be a real valued sequence such that:

$$
\sum_{\ell=0}^{K} a_{\ell}=\sum_{\ell=0}^{K} \ell a_{\ell}=0 .
$$

As an example, one can take $K=2, a_{0}=1, a_{1}=-2, a_{2}=1$.

For every $\boldsymbol{k}=\left(k_{1}, \ldots, k_{d}\right) \in \mathbb{N}^{d}, a_{\boldsymbol{k}}=\prod_{j=1}^{d} a_{k_{j}}$. Let us note

$$
\begin{aligned}
& \mathcal{K}=\left\{\boldsymbol{k} \in \mathbb{N}^{d}, 0 \leqslant k_{j} \leqslant K, j=0 \ldots K\right\}, \\
& \Delta X_{\boldsymbol{p}}(x)=\sum_{\boldsymbol{k} \in \mathcal{K}} a_{\boldsymbol{k}} X_{h}\left(\frac{\boldsymbol{k}+\boldsymbol{p}}{N}\right)=\sum_{k_{1}, \ldots, k_{d}=0}^{K} \prod_{j=1}^{d} a_{k_{j}} X_{h}\left(\frac{\boldsymbol{k}+\boldsymbol{p}}{N}\right),
\end{aligned}
$$

where $\boldsymbol{p} \in \mathcal{V}_{\varepsilon, N}(x)$, are the increments of $X_{h}$ associated with the sequence $a$.

Then the localized generalized quadratic variation at point $x$ is equal to

$$
V_{\varepsilon, N}(x)=\sum_{\boldsymbol{p} \in \mathcal{V}_{\varepsilon, N}(x)}\left(\Delta X_{\boldsymbol{p}}(x)\right)^{2} .
$$

Our aim is to show that

$$
\hat{h}_{N}(x)=\frac{1}{2}\left[\log _{2}\left(\frac{V_{\varepsilon, N / 2}(x)}{V_{\varepsilon, N}(x)}\right)+d\right]
$$

is a consistent estimator of $h(x)$.

Notations. Let $\left(v_{m}\right)_{m}$ be a deterministic real valued sequence, $\left(Z_{m}\right)_{m}$ and $\left(R_{m}\right)_{m}$ be two sequences of real random variables.

- $v_{m}=\mathrm{O}(1)$ means that the sequence $\left(v_{m}\right)_{m}$ is bounded.

- $Z_{m}=\mathrm{O}_{\mathbb{P}}(1)$ if and only if

$$
\forall \varepsilon>0, \exists M>0, \quad \sup _{m} \mathbb{P}\left(\left|Z_{m}\right|>M\right)<\varepsilon .
$$

- $Z_{m}=\mathrm{O}_{\mathbb{P}}\left(R_{m}\right)$ means $Z_{m}=R_{m} U_{m}$ with $U_{m}=\mathrm{O}_{\mathbb{P}}(1)$. 
Theorem 4.1. Let $h$ be a $\beta$-Hölder function on $[0,1]^{d}$ and suppose that $\max _{y \in[0,1]^{d}} h(y)<\beta$. Let $\varepsilon=N^{-\alpha}$ with $0<\alpha<1$, then as $N \rightarrow+\infty$

$$
\hat{h}_{N}(x) \stackrel{(\mathbb{P})}{\rightarrow} h(x)
$$

where $\stackrel{(\mathbb{P})}{\rightarrow}$ means a convergence in probability.

If $d \geqslant 2$ and $0<\alpha<1-1 / d$, then as $N \rightarrow+\infty$

$\hat{h}_{N}(x) \rightarrow h(x)$ almost surely.

Moreover for $\alpha=d /(d+2 \beta)$ and for every $\gamma<\alpha \beta$,

$$
\hat{h}_{N}(x)=h(x)+\mathrm{O}_{\mathbb{P}}\left(N^{-\min (\beta-h(x), \gamma)} \ln N\right) .
$$

Remark. Since the rate of convergence in (17) depends on $\beta$ and $h(x)$, it is only given here as a curiosity! Nevertheless we can remark that when $\beta$ is known, the last claim of Theorem 4.1 explains how to choose $\alpha$. Moreover when $\beta=1$, which is the case when $h$ is $\mathcal{C}^{1}$ for instance, it is the same choice as in [5].

In order to prove this theorem, one studies the asymptotic of $V_{\varepsilon, N}(x)$. The field

$$
\left.Y(x, y)=\int_{\mathbb{R}^{d}} \frac{\mathrm{e}^{-i x \cdot \xi}-1}{\| \xi^{d / 2+y}} L(d \xi), \quad x \in \mathbb{R}^{d}, y \in\right] 0,1[,
$$

is first introduced. Notice that $X_{h}(x)=Y(x, h(x))$ and that

$$
V_{\varepsilon, N}(x)=\sum_{\boldsymbol{p} \in \mathcal{V}_{\varepsilon, N}(x)}\left[\sum_{\boldsymbol{k} \in \mathcal{K}} a_{\boldsymbol{k}} Y\left(\frac{\boldsymbol{k}+\boldsymbol{p}}{N}, h\left(\frac{\boldsymbol{k}+\boldsymbol{p}}{N}\right)\right)\right]^{2} .
$$

Like in [3] and [1], one replaces $Y\left(\frac{\boldsymbol{k}+\boldsymbol{p}}{N}, h\left(\frac{\boldsymbol{k}+\boldsymbol{p}}{N}\right)\right)$ by $Y\left(\frac{\boldsymbol{k}+\boldsymbol{p}}{N}, h\left(\frac{\boldsymbol{p}}{N}\right)\right)$ in (18):

$$
W_{\varepsilon, N}(x)=\sum_{\boldsymbol{p} \in \mathcal{V}_{\varepsilon, N}(x)}\left[\sum_{\boldsymbol{k} \in \mathcal{K}} a_{\boldsymbol{k}} Y\left(\frac{\boldsymbol{k}+\boldsymbol{p}}{N}, h\left(\frac{\boldsymbol{p}}{N}\right)\right)\right]^{2} .
$$

The study of the asymptotic of $W_{\varepsilon, N}(x)$ is simpler than for $V_{\varepsilon, N}(x)$. Then it remains to compare $W_{\varepsilon, N}(x)$ to $V_{\varepsilon, N}(x)$. In order to obtain the asymptotic of $W_{\varepsilon, N}(x)$, one evaluates $\mathbb{E}\left(W_{\varepsilon, N}(x)\right)$ and $\operatorname{Var} W_{\varepsilon, N}(x)$. At first, let us give useful expressions of them. Please notice that because of (16),

$$
\sum_{\boldsymbol{k} \in \mathcal{K}} a_{\boldsymbol{k}} Y\left(\frac{\boldsymbol{k}+\boldsymbol{p}}{N}, h\left(\frac{\boldsymbol{p}}{N}\right)\right)=\int_{\mathbb{R}^{d}} \frac{\mathrm{e}^{-i \frac{p}{N} \cdot \xi}}{\|\xi\|^{\frac{d}{2}+h\left(\frac{\boldsymbol{p}}{N}\right)}} \sum_{\boldsymbol{k} \in \mathcal{K}} a_{\boldsymbol{k}} \mathrm{e}^{-i \frac{\boldsymbol{k}}{N} \cdot \xi} L(d \xi) .
$$

Then because of (5),

$$
\mathbb{E}\left(W_{\varepsilon, N}(x)\right)=A \sum_{\boldsymbol{p} \in \mathcal{V}_{\varepsilon, N}(x)} I_{\boldsymbol{p}, \boldsymbol{p}}
$$

where $A=\sigma^{2}+4 \pi \int_{0}^{+\infty} \rho^{2} v_{\rho}(d \rho)$ and

$$
I_{\boldsymbol{p}, \boldsymbol{p}^{\prime}}=\int_{\mathbb{R}^{d}} \mathrm{e}^{i \frac{\left(\boldsymbol{p}^{\prime}-\boldsymbol{p}\right)}{N} \cdot \xi} \frac{\left|\sum_{\boldsymbol{k} \in \mathcal{K}} a_{\boldsymbol{k}} \mathrm{e}^{-i \frac{\boldsymbol{k}}{N} \cdot \xi}\right|^{2}}{\|\xi\|^{d+h\left(\frac{\boldsymbol{p}}{N}\right)+h\left(\frac{\boldsymbol{p}^{\prime}}{N}\right)}} d \xi
$$

Moreover one can prove as it is done in the last section of [6] that

$$
\operatorname{Var} W_{\varepsilon, N}(x)=\sum_{\boldsymbol{p}, \boldsymbol{p}^{\prime} \in \mathcal{V}_{\varepsilon, N}(x)}\left[2 A^{2}\left(I_{\boldsymbol{p}, \boldsymbol{p}^{\prime}}\right)^{2}+2 B J_{\boldsymbol{p}, \boldsymbol{p}^{\prime}}+B L_{\boldsymbol{p}, \boldsymbol{p}^{\prime}}\right],
$$


where $B=4 \pi \int_{0}^{+\infty} \rho^{4} v_{\rho}(d \rho)$,

$$
J_{p, p^{\prime}}=\int_{\mathbb{R}^{d}} \frac{\left|\sum_{\boldsymbol{k} \in \mathcal{K}} a_{\boldsymbol{k}} \mathrm{e}^{-i \frac{k}{N} \cdot \xi}\right|^{4}}{\|\xi\|^{2 d+2 h\left(\frac{p}{N}\right)+2 h\left(\frac{p^{\prime}}{N}\right)}} d \xi
$$

and

$$
L_{\boldsymbol{p}, \boldsymbol{p}^{\prime}}=\int_{\mathbb{R}^{d}} \mathrm{e}^{2 i \frac{\left(p^{\prime}-p\right)}{N} \cdot \xi} \frac{\left|\sum_{\boldsymbol{k} \in \mathcal{K}} a_{\boldsymbol{k}} \mathrm{e}^{-i \frac{k}{N} \cdot \xi}\right|^{4}}{\|\xi\|^{2 d+2 h\left(\frac{p}{N}\right)+2 h\left(\frac{p^{\prime}}{N}\right)}} d \xi .
$$

Before proving Theorem 4.1, let us study $I_{p, p^{\prime}}, J_{p, p^{\prime}}$ and $L_{p, p^{\prime}}$.

Lemma 4.2. For every $\delta>0$ such that $h(x)>\delta$, there exist $\varepsilon_{0}>0$ and $N_{0} \in \mathbb{N}^{*}$ such that for $\left.\left.\varepsilon \in\right] 0, \varepsilon_{0}\right], N \geqslant N_{0}$ and $\left(\boldsymbol{p}, \boldsymbol{p}^{\prime}\right) \in \mathcal{V}_{\varepsilon, N}(x)^{2}$,

$$
\left|I_{\boldsymbol{p}, \boldsymbol{p}^{\prime}}-\frac{1}{N^{2 h(x)}} F_{2 h(x)}\left(\boldsymbol{p}^{\prime}-\boldsymbol{p}\right)\right| \leqslant \frac{C \varepsilon^{\beta} \ln N}{N^{2 \delta} \prod_{j=1}^{d}\left(1+\left|p_{j}^{\prime}-p_{j}\right|\right)},
$$

where $C$ is a nonnegative constant which only depends on $\delta$ and $x$, and where

$$
\left.F_{\gamma}(u)=\int_{\mathbb{R}^{d}} \mathrm{e}^{i u \cdot \xi} \frac{\left|\sum_{k \in \mathcal{K}} a_{\boldsymbol{k}} \mathrm{e}^{i k \cdot \xi}\right|^{2}}{\|\xi\|^{d+\gamma}} d \xi, \quad \gamma \in\right] 0,2\left[, u \in \mathbb{R}^{d} .\right.
$$

Proof. Let $r>0$ such that $\left.K_{0}=\overline{B(x, r)} \subset\right] 0,1\left[{ }^{d}\right.$ and $m=\min _{K_{0}} h>\delta$. Let $M=\max _{K_{0}} h$. Then let $\varepsilon_{0}>0$ and $N_{0} \in \mathbb{N}^{*}$ such that for every $\boldsymbol{p} \in \mathcal{V}_{\varepsilon, N}(x)$ and $\boldsymbol{k}$,

$$
\frac{\boldsymbol{k}+\boldsymbol{p}}{N} \in K_{0} \text {. }
$$

Let us remark that

$$
I_{\boldsymbol{p}, \boldsymbol{p}^{\prime}}=\frac{1}{N^{h\left(\frac{p}{N}\right)+h\left(\frac{p^{\prime}}{N}\right)}} F_{h\left(\frac{p}{N}\right)+h\left(\frac{p^{\prime}}{N}\right)}\left(\boldsymbol{p}^{\prime}-\boldsymbol{p}\right) .
$$

Notice that for every $u \in \mathbb{R}^{d}$, the map $\gamma \mapsto \frac{1}{N^{\gamma}} F_{\gamma}(u)$ is $\mathcal{C}^{1}$ on ]0, 2[. Then a Taylor expansion gives:

$$
\left|I_{\boldsymbol{p}, \boldsymbol{p}^{\prime}}-\frac{1}{N^{2 h(x)}} F_{2 h(x)}\left(\boldsymbol{p}^{\prime}-\boldsymbol{p}\right)\right| \leqslant C \varepsilon^{\beta} \max _{2 m \leqslant \gamma \leqslant 2 M}\left|A\left(\boldsymbol{p}, \boldsymbol{p}^{\prime}, \gamma\right)\right|,
$$

where $A\left(\boldsymbol{p}, \boldsymbol{p}^{\prime}, \gamma\right)=\frac{1}{N^{\gamma}} A_{1}\left(\boldsymbol{p}, \boldsymbol{p}^{\prime}, \gamma\right)+\frac{\ln N}{N^{\gamma}} F_{\gamma}\left(\boldsymbol{p}^{\prime}-\boldsymbol{p}\right)$,

$$
\text { with } \quad A_{1}\left(\boldsymbol{p}, \boldsymbol{p}^{\prime}, \gamma\right)=\int_{\mathbb{R}^{d}} \frac{\ln \|\xi\| \mathrm{e}^{i\left(\boldsymbol{p}^{\prime}-\boldsymbol{p}\right) \cdot \xi}\left|\sum_{\boldsymbol{k} \in \mathcal{K}} a_{\boldsymbol{k}} \mathrm{e}^{i \boldsymbol{k} \cdot \xi}\right|^{2}}{\|\xi\|^{d+\gamma}} d \xi .
$$

Notice that $A(\boldsymbol{p}, \boldsymbol{p}, \gamma)=A(\gamma)$. Then suppose that $\boldsymbol{p} \neq \boldsymbol{p}^{\prime}$ and proceed like [6] or [7] by integrating by parts, which leads to

$$
\left|A_{1}\left(\boldsymbol{p}, \boldsymbol{p}^{\prime}, \gamma\right)\right| \leqslant C \prod_{\left\{j / p_{j} \neq p_{j^{\prime}}\right\}} \frac{1}{\left|p_{j}^{\prime}-p_{j}\right|} \leqslant C \prod_{j=1}^{d} \frac{1}{1+\left|p_{j}^{\prime}-p_{j}\right|} .
$$


Moreover one can also prove, using integrations by part that

$$
\max _{2 m \leqslant \gamma \leqslant 2 M}\left|F_{\gamma}\left(\boldsymbol{p}^{\prime}-\boldsymbol{p}\right)\right| \leqslant C \prod_{j=1}^{d} \frac{1}{1+\left|p_{j}^{\prime}-p_{j}\right|} .
$$

Then since $m>\delta$,

$$
\left|I_{\boldsymbol{p}, \boldsymbol{p}^{\prime}}-\frac{1}{N^{2 h(x)}} F_{2 h(x)}\left(\boldsymbol{p}^{\prime}-\boldsymbol{p}\right)\right| \leqslant C \frac{\varepsilon^{\beta} \ln N}{N^{2 \delta}} \prod_{j=1}^{d} \frac{1}{1+\left|p_{j}^{\prime}-p_{j}\right|} .
$$

Lemma 4.3. For every $\delta>0$ such that $h(x)>\delta$, there exist $\varepsilon_{0}>0, N_{0} \in \mathbb{N}^{*}$ such that for $\left.\left.\varepsilon \in\right] 0, \varepsilon_{0}\right], N \geqslant N_{0}$ and $\left(\boldsymbol{p}, \boldsymbol{p}^{\prime}\right) \in \mathcal{V}_{\varepsilon, N}(x)^{2}$,

$$
\left|J_{\boldsymbol{p}, \boldsymbol{p}^{\prime}}\right| \leqslant C\left[\frac{1}{N^{d+4 h(x)}}+\frac{\varepsilon^{\beta} \ln N}{N^{d+4 \delta}}\right],
$$

where $C$ is a nonnegative constant which only depends on $\delta$ and $x$.

The same inequality holds for $L_{p, p^{\prime}}$.

Proof. Notice that

$$
\left|L_{\boldsymbol{p}, \boldsymbol{p}^{\prime}}\right| \leqslant J_{\boldsymbol{p}, \boldsymbol{p}^{\prime}}
$$

Therefore it is sufficient to prove the lemma for $J_{\boldsymbol{p}, \boldsymbol{p}^{\prime}}$. Let $K_{0}=\overline{B(x, r)}, \varepsilon_{0}$ and $N_{0}$ be such as in the proof of the previous lemma.

Please notice that

$$
J_{\boldsymbol{p}, \boldsymbol{p}^{\prime}}=\frac{1}{N^{d+2 h\left(\frac{p}{N}\right)+2 h\left(\frac{p^{\prime}}{N}\right)}} G_{2 h\left(\frac{p}{N}\right)+2 h\left(\frac{p^{\prime}}{N}\right)}(0),
$$

where

$$
G_{\gamma}(u)=\int_{\mathbb{R}^{d}} \mathrm{e}^{-2 i u \cdot \xi} \frac{\left|\sum_{\boldsymbol{k} \in \mathcal{K}} a_{\boldsymbol{k}} \mathrm{e}^{i \boldsymbol{k} \cdot \xi}\right|^{4}}{\|\xi\|^{2 d+\gamma}} d \xi .
$$

Since the map $\gamma \mapsto \frac{1}{N^{\gamma}} G_{\gamma}(0)$ is $\mathcal{C}^{1}$ on $] 0,4[$, a Taylor expansion leads to

$$
\left|J_{\boldsymbol{p}, \boldsymbol{p}^{\prime}}-\frac{1}{N^{d+4 h(x)}} G_{4 h(x)}(0)\right| \leqslant \frac{C \varepsilon^{\beta} \ln N}{N^{d+4 \delta}} .
$$

Let us now prove Theorem 4.1 .

Proof of Theorem 4.1. Let us take $\varepsilon=N^{-\alpha}$ with $\left.\alpha \in\right] 0,1[$. Let $\delta>0$ such that $\delta<h(x)$.

Step 1. Asymptotic of $W_{\varepsilon, N}$. One deduces from equality (20) and from Lemma 4.2 that

$$
\mathbb{E}\left(W_{\varepsilon, N}(x)\right)=\varepsilon^{d} N^{d-2 h(x)} D(x)+\mathrm{O}\left(N^{-2 h(x)}\right)+\mathrm{O}\left(\varepsilon^{d+\beta} N^{d-2 \delta} \ln N\right),
$$

where $D(x)=2^{d} A F_{2 h(x)}(0)$.

It remains to study $\operatorname{Var} W_{\varepsilon, N}(x)$. In the sequel Lemmas 4.2 and 4.3 are applied in order to estimate this variance. Let us first recall that

$$
\left|F_{2 h(x)}\left(\boldsymbol{p}^{\prime}-\boldsymbol{p}\right)\right| \leqslant C \prod_{j=1}^{d} \frac{1}{1+\left|p_{j}^{\prime}-p_{j}\right|},
$$


and remark that

$$
\sum_{\boldsymbol{p}, \boldsymbol{p}^{\prime} \in \mathcal{V}_{\varepsilon, N}(x)} \prod_{j=1}^{d} \frac{1}{\left(1+\left|p_{j}^{\prime}-p_{j}\right|\right)^{2}} \leqslant C \varepsilon^{d} N^{d} .
$$

Then by applying Lemmas 4.2 and 4.3 to (22) with $\delta>h(x)-\alpha \beta / 2$,

$$
\operatorname{Var} W_{\varepsilon, N}(x)=\mathrm{O}\left(\varepsilon^{d} N^{d-4 h(x)}\right) \text {. }
$$

Consequently, if $d(1-\alpha)>1$, then by Borel-Cantelli, as $N \rightarrow+\infty$,

$$
\varepsilon^{-d} N^{-d+2 h(x)} W_{\varepsilon, N}(x) \rightarrow D(x) \text { almost surely. }
$$

Furthermore, because of (25) and (26),

$$
W_{\varepsilon, N}(x)=\varepsilon^{d} N^{d-2 h(x)} D(x)+\mathrm{O}_{\mathbb{P}}\left(\varepsilon^{d / 2} N^{d / 2-2 h(x)}\right)+\mathrm{O}\left(\varepsilon^{d+\beta} N^{d-2 \delta} \ln N\right) .
$$

At first, suppose that $\alpha \leqslant d /(d+2 \beta)$, then since $\delta<h(x)$,

$$
(d+\beta) \alpha-d+2 \delta<d(\alpha-1) / 2+2 h(x) .
$$

Consequently, since $\varepsilon=N^{-\alpha}$,

$$
W_{\varepsilon, N}(x)=\varepsilon^{d} N^{d-2 h(x)} D(x)+\mathrm{O}_{\mathbb{P}}\left(\varepsilon^{d+\beta} N^{d-2 \delta} \ln N\right) .
$$

Else suppose that $\alpha>d /(d+2 \beta)$, then one can choose

$$
\delta>h(x)-(d+2 \beta) \alpha / 4+d / 4 .
$$

For this choice of $\delta$,

$$
W_{\varepsilon, N}(x)=\varepsilon^{d} N^{d-2 h(x)} D(x)+\mathrm{O}_{\mathbb{P}}\left(\varepsilon^{d / 2} N^{d / 2-2 h(x)}\right) \text {. }
$$

Step 2. Comparison of $W_{\varepsilon, N}(x)$ and $V_{\varepsilon, N}(x)$. Since $Y$ may have infinite moments, it is split into $Y=Y_{n}^{+}+Z_{n}$ where $n \geqslant d / 2$ and $Z_{n}$ is defined by (12). Notice that

$$
X_{h, n}^{+}(y)=Y_{n}^{+}(y, h(y)) \quad \text { and that } \quad X_{h, n}^{-}(y)=Z_{n}(y, h(y)) .
$$

Moreover

$$
\left|V_{\varepsilon, N}^{1 / 2}(x)-W_{\varepsilon, N}^{1 / 2}(x)\right|^{2} \leqslant 2 A(\varepsilon, N)+2 B(\varepsilon, N)
$$

where

$$
A(\varepsilon, N)=\sum_{\boldsymbol{p} \in \mathcal{V}_{\varepsilon, N}(x)}\left[\sum_{\boldsymbol{k} \in \mathcal{K}} a_{\boldsymbol{k}}\left(X_{h, n}^{+}\left(\frac{\boldsymbol{k}+\boldsymbol{p}}{N}\right)-Y_{n}^{+}\left(\frac{\boldsymbol{k}+\boldsymbol{p}}{N}, h\left(\frac{\boldsymbol{p}}{N}\right)\right)\right)\right]^{2}
$$

and

$$
B(\varepsilon, N)=\sum_{\boldsymbol{p} \in \mathcal{V}_{\varepsilon, N}(x)}\left[\sum_{\boldsymbol{k} \in \mathcal{K}} a_{\boldsymbol{k}}\left(X_{h, n}^{-}\left(\frac{\boldsymbol{k}+\boldsymbol{p}}{N}\right)-Z_{n}\left(\frac{\boldsymbol{k}+\boldsymbol{p}}{N}, h\left(\frac{\boldsymbol{p}}{N}\right)\right)\right)\right]^{2} .
$$

Since $Z_{n}$ has $\mathcal{C}^{1}$-sample paths,

$$
|B(\varepsilon, N)| \leqslant C \varepsilon^{d} N^{d-2 \beta},
$$

where $C$ is a random variable.

Let us now study the moments of $A(\varepsilon, N)$. Notice that

$$
X_{h, n}^{+}\left(\frac{\boldsymbol{k}+\boldsymbol{p}}{N}\right)-Y_{n}^{+}\left(\frac{\boldsymbol{k}+\boldsymbol{p}}{N}, h\left(\frac{\boldsymbol{p}}{N}\right)\right)=\int_{\mathbb{R}^{d}} g_{n, 2}\left(\frac{\boldsymbol{k}+\boldsymbol{p}}{N}, \frac{\boldsymbol{p}}{N}, \xi\right) L(d \xi),
$$


where $g_{n, 2}$ is defined by (11). Then by applying Proposition 3.1 and Lemma 3.3, one obtains that

$$
\mathbb{E}\left(A(\varepsilon, N)^{q}\right) \leqslant C \varepsilon^{d q} N^{q(d-2 \beta)} .
$$

Then since $\beta>h(x)$, one can choose $q \in \mathbb{N}^{*}$ such that $2 q(\beta-h(x))>1$. Borel-Cantelli leads to:

$$
\varepsilon^{-d} N^{-d+2 h(x)} A(\varepsilon, N) \rightarrow 0 \quad \text { almost surely, as } N \rightarrow+\infty \text {. }
$$

Moreover $A(\varepsilon, N)=\mathrm{O}_{\mathbb{P}}\left(\varepsilon^{d} N^{d-2 \beta}\right)$. Consequently,

$$
V_{\varepsilon, N}^{1 / 2}(x)-W_{\varepsilon, N}^{1 / 2}(x)=\mathrm{O}_{\mathbb{P}}\left(\varepsilon^{d / 2} N^{d / 2-\beta}\right)
$$

and as $N \rightarrow+\infty$

$$
\varepsilon^{-d / 2} N^{-d / 2+h(x)}\left(V_{\varepsilon, N}^{1 / 2}(x)-W_{\varepsilon, N}^{1 / 2}(x)\right) \rightarrow 0 \quad \text { almost surely. }
$$

Step 3. Conclusion. Notice that

$$
V_{\varepsilon, N}(x)=W_{\varepsilon, N}(x)+2 W_{\varepsilon, N}^{1 / 2}(x) D_{\varepsilon, N}(x)+D_{\varepsilon, N}^{2}(x),
$$

where $D_{\varepsilon, N}(x)=\left(V_{\varepsilon, N}^{1 / 2}(x)-W_{\varepsilon, N}^{1 / 2}(x)\right)$. Then when $d(1-\alpha)>1$, because of (27) and (32), and since $A F_{2 h(x)}(0) \neq 0$,

$$
\hat{h}_{N}(x) \rightarrow h(x) \quad \text { almost surely as } N \rightarrow+\infty .
$$

Even if we do not need the asymptotic of $V_{\varepsilon, N}(x)$ for every $\alpha$ to prove the theorem, let us state it. Using equalities (28), (29) and (31), one can prove:

- if $\alpha>d /(d+2 \beta)$, then

○ if $h(x) \leqslant \beta-d(1-\alpha) / 2$,

$$
V_{\varepsilon, N}(x)=2^{d} A \varepsilon^{d} N^{d-2 h(x)} F_{2 h(x)}(0)+\mathrm{O}_{\mathbb{P}}\left(\varepsilon^{d / 2} N^{d / 2-2 h(x)}\right),
$$

○ else

$$
V_{\varepsilon, N}(x)=2^{d} A \varepsilon^{d} N^{d-2 h(x)} F_{2 h(x)}(0)+\mathrm{O}_{\mathbb{P}}\left(\varepsilon^{d} N^{d-h(x)-\beta}\right) ;
$$

- if $\alpha \leqslant d /(d+2 \beta)$, then

○ if $h(x) \geqslant \beta-\alpha \beta$,

$$
V_{\varepsilon, N}(x)=2^{d} A \varepsilon^{d} N^{d-2 h(x)} F_{2 h(x)}(0)+\mathrm{O}_{\mathbb{P}}\left(\varepsilon^{d} N^{d-h(x)-\beta}\right),
$$

- else

$$
V_{\varepsilon, N}(x)=2^{d} A \varepsilon^{d} N^{d-2 h(x)} F_{2 h(x)}(0)+\mathrm{O}_{\mathbb{P}}\left(\varepsilon^{d+\beta} N^{d-2 \delta} \ln N\right)
$$

where $\delta<h(x)$.

Let us now take $\alpha=d /(d+2 \beta)$ and explain in few words this choice. Let us first recall that

$$
W_{\varepsilon, N}(x)=\varepsilon^{d} N^{d-2 h(x)} D(x)+\mathrm{O}_{\mathbb{P}}\left(\varepsilon^{d / 2} N^{d / 2-2 h(x)}\right)+\mathrm{O}\left(\varepsilon^{d+\beta} N^{d-2 \delta} \ln N\right) .
$$

For a given $\delta$, since $\varepsilon=N^{-\alpha}$, the best choice of $\alpha$ is obtained by solving the following equation in $\alpha$ :

$$
\frac{-\alpha d+d}{2}-2 h(x)=-(d+\beta) \alpha+d-2 \delta
$$

which gives

$$
\alpha=\frac{d-4 \delta+4 h(x)}{d+2 \beta} .
$$


Then the two errors terms are of the same order up to a logarithmic factor. But since the error term is a nonincreasing function of $\delta$ and since $\delta$ can be arbitrarily chosen closed to $h(x)$, one chooses

$$
\alpha=\frac{d}{d+2 \beta} .
$$

In this case,

$$
V_{\varepsilon, N}(x)=2^{d} A \varepsilon^{d} N^{d-2 h(x)} F_{2 h(x)}(0)+\mathrm{O}_{\mathbb{P}}\left(N^{-\eta(x)} \ln N\right),
$$

where

$$
\eta(x)=\min ((d+\beta) \alpha-d+2 \delta, d \alpha-d+h(x)+\beta) .
$$

Then using Lemma 2.12 of [15], one can prove that

$$
\log _{2} V_{\varepsilon, N}(x)=\log _{2}\left(2^{d} A \varepsilon^{d} N^{d-2 h(x)} F_{2 h(x)}(0)\right)+\mathrm{O}_{\mathbb{P}}\left(N^{-\min (\beta \alpha+2 \delta-2 h(x), \beta-h(x))} \ln N\right),
$$

and conclude that

$$
\hat{h}_{N}(x)=h(x)+\mathrm{O}_{\mathbb{P}}\left(N^{-\min (\beta \alpha+2 \delta-2 h(x), \beta-h(x))} \ln N\right) .
$$

Notice that with the same argument one can give a rate of convergence for every choice of $\alpha$.

\section{References}

[1] A. Ayache, A. Benassi, S. Cohen, J. Lévy Véhel, Regularity and identification of generalized multifractional gaussian process, Séminaire de Probabilités (2004), submitted for publication.

[2] A. Ayache, J. Lévy Véhel, The generalized multifractional Brownian motion, Stat. Inference Stoch. Process. 3 (1-2) (2000) 7-18, 19th Rencontres Franco-Belges de Statisticiens, Marseille, 1998.

[3] A. Ayache, J. Lévy Véhel, Identification de l'exposant de Hölder ponctuel d'un Mouvement Brownien Multifractionnaire Généralisé, Technical Report LSP-2002-02, Laboratoire de Statistique et de Probabilités, UMR C5583, Université Paul Sabatier, 2002.

[4] A. Benassi, P. Bertrand, S. Cohen, J. Istas, Identification of the Hurst index of a step fractional Brownian motion, Stat. Inference Stoch. Process. 3 (1-2) (2000) 101-111, 19th Rencontres Franco-Belges de Statisticiens, Marseille, 1998.

[5] A. Benassi, S. Cohen, J. Istas, Identifying the multifractional function of a Gaussian process, Statist. Probab. Lett. 39 (1998) $337-345$.

[6] A. Benassi, S. Cohen, J. Istas, Identification and properties of real harmonizable fractional Lévy motions, Bernoulli 8 (1) (2002) 97-115.

[7] A. Benassi, S. Cohen, J. Istas, S. Jaffard, Identification of filtered white noises, Stoch. Process. Appl. 75 (1998) 31-49.

[8] A. Benassi, S. Jaffard, D. Roux, Gaussian processes and pseudodifferential elliptic operators, Revista Mathematica Iberoamericana 13 (1) (1997) 19-89.

[9] H. Cramér, M.R. Leadbetter, Stationary and Related Stochastic Processes. Sample Function Properties and Their Applications, Wiley, New York, 1967.

[10] J. Istas, G. Lang, Quadratic variations and estimation of the local Holder index of a gaussian process, Ann. Inst. Poincaré 33 (4) (1997) 407-437.

[11] S. Mallat, A theory for multiresolution signal decomposition: the wavelet representation, IEEE Trans. PAMI 11 (1989) 674-693.

[12] B. Mandelbrot, J.V. Ness, Fractional brownian motion, fractionnal noises and applications, Siam Rev. 10 (1968) $422-437$.

[13] R. Peltier, J. Lévy Véhel, Multifractional Brownian motion: definition and preliminary results, available on http://wwwsyntim.inria.fr/fractales/, 1996.

[14] G. Samorodnitsky, M.S. Taqqu, Stable Non-Gaussian Random Processes, Chapman \& Hall, New York, 1994.

[15] A.W. van der Vaart, Asymptotic Statistics, Cambridge University Press, Cambridge, 1998.

[16] B. Vidakovic, Statistical Modeling by Wavelets, Wiley, New York, 1999, a Wiley-Interscience Publication. 